\title{
Rapid rewarming after therapeutic hypothermia worsens outcome in sepsis
}

\section{You Hwan Jo', Kyuseok Kim¹, Jae Hyuk Lee', Kwang Pil Rim², In $\mathrm{Soo} \mathrm{Cho}^{3}$}

'Department of Emergency Medicine, Seoul National University Bundang Hospital, Seongnam, Korea ${ }^{2}$ Department of Emergency Medicine, St. Carollo General Hospital, Suncheon, Korea

${ }^{3}$ Department of Emergency Medicine, Kepco Medical Center, Seoul, Korea

Objective This study was performed to investigate the effect of the rewarming rate on survival and acute lung injury in sepsis.

Methods Male Sprague-Dawley rats underwent cecal ligation and incision. After 1 hour of sepsis induction, normothermia $\left(37^{\circ} \mathrm{C} \pm 0.5^{\circ} \mathrm{C}\right.$, NT group) or hypothermia $\left(32^{\circ} \mathrm{C} \pm 0.5^{\circ} \mathrm{C}\right)$ was induced. Hypothermia was maintained for 4 hours and rats were divided into two groups according to the rewarming rate: RW1 group, 1 hour of rewarming; and RW2 group, 2 hours of rewarming. In the survival study, rats were observed for 12 hours after sepsis induction ( $n=6$ per group). In the second experiment, rats were sacrificed 7 hours after sepsis induction, and lung tissues and plasma were harvested ( $n=10$ per group).

Results In the survival study, the RW2 group survived longer than the RW1 group $(P<0.05)$, but the RW1 and NT groups showed no significant difference in survival duration $(P>0.05)$. The histological lung injury score and malondialdehyde concentrations in the lung tissues were significantly higher in the RW1 group than in the RW2 group $(P<0.05)$. Plasma interleukin (IL)- 6 concentration and the ratio of IL-6 to IL-10 were higher in the RW1 group than in the RW2 group $(\mathrm{P}<0.05)$.

Conclusion Rapid rewarming after therapeutic hypothermia results in a shorter survival period and acute lung injury in sepsis, which could be associated with the inflammatory responses.

Keywords Hypothermia; Rewarming; Sepsis; Survival; Lung injury

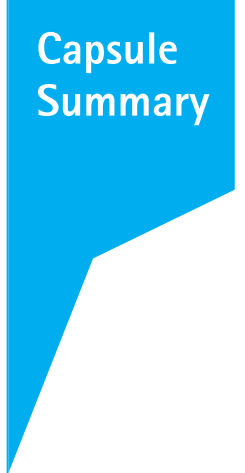

What is already known

The effect of therapeutic hypothermia on sepsis is under debate and it depends on the model of sepsis, time of application, rewarming and the severity of sepsis.

What is new in the current study

Rapid rewarming after therapeutic hypothermia shortens survival duration and worsens acute lung injury compared to slow rewarming.
Received: 30 June 2014

Revised: 5 July 2014

Accepted: 17 July 2014

Correspondence to: Kyuseok Kim Department of Emergency Medicine, Seoul National University Bundang Hospital, 166 Gumi-ro, Bundang-gu, Seongnam 463-707, Korea

E-mail:dremkks@snubh.org

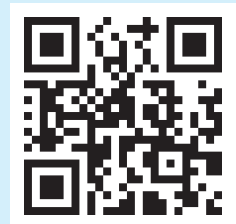

How to cite this article:

Jo YH, Kim K, Lee JH, Rim KP, Cho IS. Rapid rewarming after therapeutic hypothermia worsens outcome in sepsis. Clin Exp Emerg Med 2014;1(2):120-125.

This is an Open Access article distributed under the terms of the Creative Commons Attribution Non-Commercial License (http:// creativecommons.org/licenses/by-nc/3.0/). 


\section{INTRODUCTION}

Therapeutic hypothermia $(\mathrm{TH})$ is currently recommended for comatose patients after cardiac arrest. 'In addition to cardiac arrest, use of TH has been evaluated in various clinical and experimental conditions such as perinatal asphyxia, traumatic brain injury, stroke, myocardial infarction, hemorrhagic shock, and ischemiareperfusion injury. ${ }^{2-5}$

Rewarming is an essential process following $\mathrm{TH}$. The current recommendations are to rewarm slowly and avoid fever. ${ }^{6}$ However, few studies have been performed to analyze the optimal rate and method of rewarming. Several studies have shown that rapid rewarming worsens outcomes including survival rate and tissue injury. ${ }^{5,7}$ During the rewarming phase after $\mathrm{TH}$, the inflammatory response may be altered in patients who experienced cardiac arrest ${ }_{1}^{9}$ and the detrimental effect of rapid rewarming has been reported to be associated with the inflammatory response and intracellular calcium concentration. ${ }^{5,8,10,11}$

The incidence of sepsis and resulting mortality rates are high despite improved therapeutic strategies. Studies on TH in sepsis have reported beneficial effects associated with the modulation of the inflammatory response. ${ }^{12,13}$ In contrast, TH has also been reported to increase mortality and tissue injury in several sepsis models. ${ }^{14,15}$ Therefore, the effect of TH on sepsis is debatable.

We previously reported that the effect of $\mathrm{TH}$ on sepsis varies according to the severity of sepsis and that TH had a protective effect only in a model of severe sepsis. ${ }^{16}$ The inflammatory response is an important element in the pathophysiology of sepsis, and rapid rewarming could induce the inflammatory burst after TH. Therefore, we performed this study to investigate the effect of the rewarming rate following $\mathrm{TH}$ on survival rate and acute lung injury in a cecal ligation and incision model of sepsis. We hypothesized that rapid rewarming after $\mathrm{TH}$ would worsen outcomes in a severe sepsis model.

\section{METHODS}

\section{Experimental animals}

This study was approved by the Institutional Animal Care and Use Committee of Seoul National University Bundang Hospital in accordance with National Institutes of Health Guidelines. Male Sprague-Dawley rats weighing 300-350 g were used for the experiments. Animals were housed in a controlled environment with free access to food and water for 1 week before the experiment.

\section{Induction of sepsis}

Animals were anesthetized with an intramuscular injection of Zoletil (50 mg/kg, Virbac, Carros, France) and xylazine (10 mg/kg, Bayer, Seoul, Korea). Sepsis was induced by cecal ligation and incision as previously described. ${ }^{16,17}$ Briefly, a 2-cm midline incision of the abdomen was performed and the cecum was extruded. The distal one-third of the cecum was ligated, and a $0.5-\mathrm{cm}$ incision was made on the anti-mesenteric surface of the ligated cecum. The cecum was gently squeezed to expel feces into the peritoneal cavity, and $20 \mathrm{~mL} / \mathrm{kg}$ of $0.9 \%$ saline solution was then infused into the peritoneal cavity to distribute the feces evenly. The incised abdominal wall was then closed, and $30 \mathrm{~mL} / \mathrm{kg}$ of $0.9 \%$ saline solution was administered subcutaneously for fluid resuscitation.

\section{Experimental groups and protocols}

Rats were randomly divided into one of the three groups: normothermia (NT) group; 1 hour of rewarming after 4 hours of hypothermia (RW1) group; and 2 hours of rewarming after 4 hours of hypothermia (RW2) group. We monitored the rectal temperature of the rats (Harvard Homeothermic Monitor, Boston, MA, USA) throughout the experiment. One hour after sepsis induction, NT $\left(37^{\circ} \mathrm{C} \pm 0.5^{\circ} \mathrm{C}\right)$ was maintained using a heating lamp and pad. Hypothermia was induced by surface cooling with ice packs, and the target temperature $\left(32^{\circ} \mathrm{C} \pm 0.5^{\circ} \mathrm{C}\right)$ was reached within 15 minutes and maintained using a cooling pad for 4 hours. The rats were then rewarmed to $37^{\circ} \mathrm{C}$ for 1 hour in the RW1 group and 2 hours in the RW2 group using a heating lamp and pad.

In the survival study, rats were observed for 12 hours after induction of sepsis ( $n=6$ in each group). In the second experiment, rats were sacrificed 7 hours after the induction of sepsis $(n=10$ in each group). We performed laparotomy and withdrew blood samples from the abdominal aorta. The blood was then centrifuged for 15 minutes at $3,000 \mathrm{rpm}$ at $4^{\circ} \mathrm{C}$, and the separated plasma was stored at $-70^{\circ} \mathrm{C}$ for subsequent analyses. We also harvested lung tissues, and the right lower lobe of the lung was fixed with $4 \%$ formaldehyde solution for histological examination. The remaining lung tissues were stored at $-70^{\circ} \mathrm{C}$ for subsequent analyses.

\section{Histological acute lung injury score}

The right lower lobe of the lung was embedded in paraffin and the paraffin blocks were cut into 4-mm sections. The sections were deparaffinized and stained with hematoxylin and eosin. A board-certified pathologist blinded to the study groups scored the acute lung injury according to the following 4 criteria: alveolar congestion, hemorrhage, infiltration of neutrophils in the air spaces or vessel walls, and the thickness of alveolar wall/hyaline 
membrane formation. ${ }^{18}$ The score for each criterion ranged from 0 (minimal) to 4 (severe), and the sum of the scores was calculated ranging from 0 to 16.

\section{Malondialdehyde concentration in lung tissue}

Malondialdehyde (MDA) is a by-product of lipid peroxidation and an oxidative stress marker. MDA level was measured using Ohkawa's method with thiobarbituric acid and presented in $\mathrm{nmol} / \mathrm{g}$ of tissue. ${ }^{19}$

\section{Plasma interleukin-6 and interleukin-10 concentration}

Plasma interleukin (IL)-6 and IL-10 concentrations were measured with enzyme-linked immunosorbent assay kits (R\&D Systems Inc., Minneapolis, MN, USA). The concentrations are presented as $\mathrm{pg} / \mathrm{mL}$.

\section{Statistical analyses}

The survival rates were assessed with Kaplan-Meier curves and the log-rank test. Data are presented as mean \pm standard error of the mean (SE) and Student t-test was performed to compare the variables. Statistical analyses were performed with SPSS ver. 15.0 (SPSS Inc., Chicago, IL, USA) and a P-value $<0.05$ was considered statistically significant.

\section{RESULT}

\section{Survival rates}

All rats died within 12 hours of sepsis induction (Fig. 1). The median survival duration of the NT, RW1, and RW2 groups was 6.8,

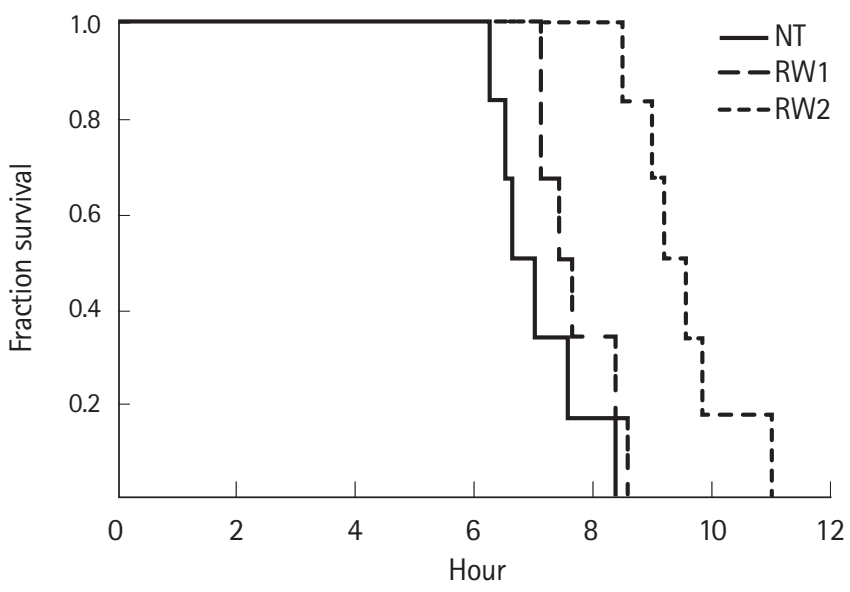

Fig. 1. Effect of the rewarming rate after therapeutic hypothermia on survival. The RW2 group survived significantly longer than the NT and RW1 groups $(P<0.05)$. There is no difference in survival duration between the NT group and RW1 group ( $P>0.05)$. NT, normothermia; RW1, 1 hour of rewarming after 4 hours of therapeutic hypothermia; RW2, 2 hours of rewarming after 4 hours of therapeutic hypothermia. $n=6$ per group.
7.6, and 9.4 hours, respectively. The survival duration of the RW2 group was significantly longer than that of the NT and RW1 groups $(P<0.001$ and $P=0.002$, respectively). However, there was no significant difference in survival duration between the NT and RW1 groups $(P=0.213)$.

In the survival study, 4 of 6 rats in the NT group died within 7 hours of sepsis induction. Therefore, the subsequent measurements of tissue injury and plasma cytokines were performed only in the RW1 and RW2 groups, not in the NT group.

\section{Histological acute lung injury score}

The acute lung injury scores of the RW1 and the RW2 groups were $8.4 \pm 0.3$ and $7.0 \pm 0.4$, respectively, and the acute lung injury score of the RW1 group was significantly higher than that of the RW2 group ( $P=0.038)$ (Fig. 2).

\section{MDA concentration in the lung tissue}

The MDA concentrations in the RW1 and RW2 groups were $83.8 \pm 3.9 \mathrm{nmol} / \mathrm{g}$ and $73.4 \pm 2.7 \mathrm{nmol} / \mathrm{g}$, respectively (Fig. 3). The difference between the two groups was statistically significant $(P=0.039)$.

\section{Plasma IL-6 and IL-10 concentrations}

The plasma concentration of IL- 6 was significantly higher in the RW1 group than in the RW2 group $(3,072 \pm 281.4 \mathrm{pg} / \mathrm{mL}$ vs. $1,865 \pm 410.5 \mathrm{pg} / \mathrm{mL} ; \mathrm{P}=0.026$ ) (Fig. 4A). The IL-10 concentration did not differ significantly between the RW2 group and the RW1 group (352.6 $\pm 48.8 \mathrm{pg} / \mathrm{mL}$ vs. $301.0 \pm 36.9 \mathrm{pg} / \mathrm{mL} ; \mathrm{P}=0.443$ ) (Fig. $4 B)$. The ratio of IL- 6 to IL-10 was $8.8 \pm 1.1$ in the RW1 group and $3.9 \pm 1.1$ in the RW2 group; it was significantly higher in the RW1

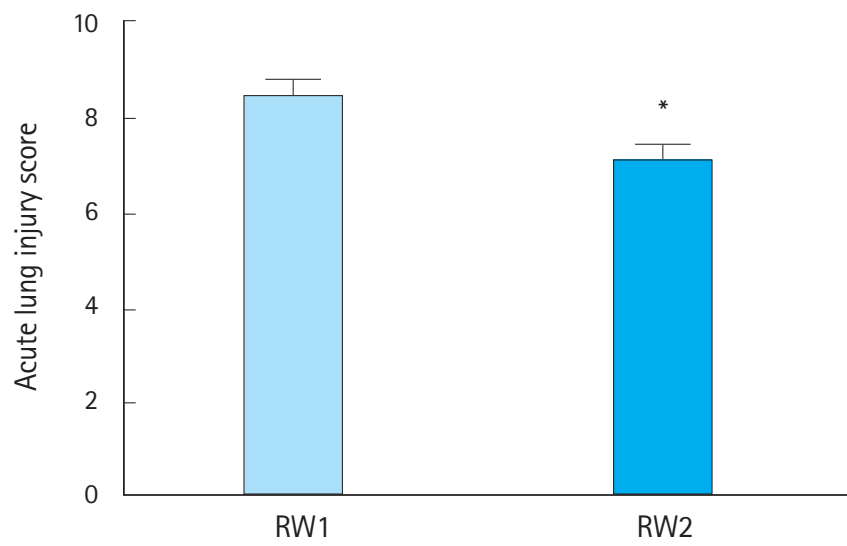

Fig. 2. Histologic lung injury. The acute lung injury score is significantly higher in the RW1 group than in the RW2 group. RW1, 1 hour of rewarming after 4 hours of therapeutic hypothermia; RW2, 2 hours of rewarming after 4 hours of therapeutic hypothermia. Values are presented as mean \pm SE. $n=10$ per group. ${ }^{*} P<0.05$. 


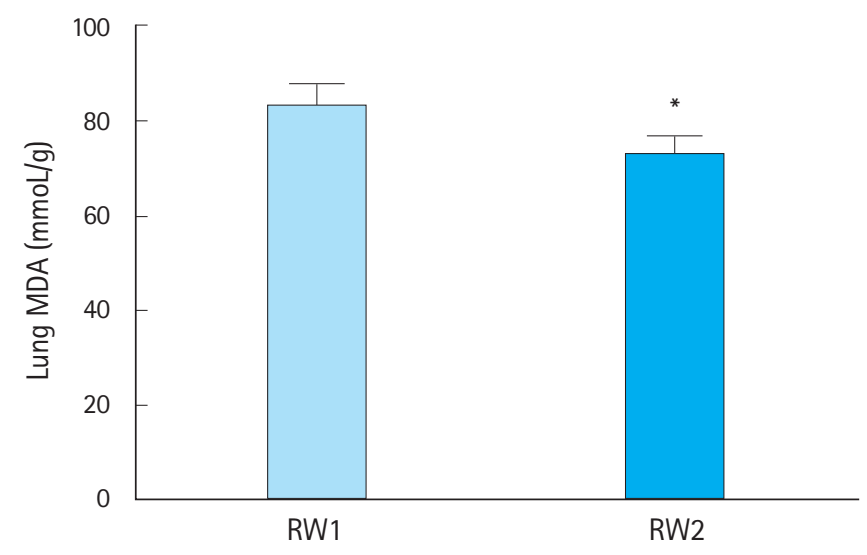

Fig. 3. Malondialdehyde (MDA) concentrations in the lung tissue. The lung MDA concentration is higher in the RW1 group than in the RW2 group. RW1, 1 hour of rewarming after 4 hours of therapeutic hypothermia; RW2, 2 hours of rewarming after 4 hours of therapeutic hypothermia. Values are presented as mean \pm SE. $n=10$ per group. ${ }^{*} \mathrm{P}<0.05$.

group than in the RW2 group ( $P=0.010)$ (Fig. 4C).

\section{DISCUSSION}

The effect of rewarming after TH has been studied in various situations. In a hemorrhagic shock model, rewarming was found to lower blood pressure and increase heart rate and the synthesis of reactive oxygen species (ROS). ${ }^{10}$ Rewarming has also been reported to induce derangement of cerebrovascular activity in patients with traumatic brain injur..$^{20}$ In cardiac arrest patients, the inflammatory cytokines and complement and adhesion molecules changed during the $\mathrm{TH}$ and rewarming phases. ${ }^{9}$ In addition, the effect of rewarming and its rate has been studied. Rapid rewarming has been reported to influence the survival rate in hemorrhagic shock and induce secondary axonal injury in traumatic brain injury. ${ }^{7.21}$ During cardiopulmonary bypass, rapid rewarming has been reported to result in deterioration of neurocognitive function and brain injury. ${ }^{8.22}$ In an intestinal ischemia-reperfusion model, gradual rewarming was found to improve the survival rate and attenuate injury to the lungs. ${ }^{5}$ In the present study, our results showed that rapid rewarming shortens survival duration and aggravates acute lung injury in a severe sepsis model.

Since $\mathrm{TH}$ has been studied in various clinical and experimental models, several studies have evaluated the effect of TH on sepsis, but the results have been inconsistent. We previously reported that TH had a beneficial effect only in a severe sepsis model and that it might be associated with suppression of the exacerbated inflammatory responses. ${ }^{16}$ However, in the present study, rapid rewarming worsened both survival rate and acute lung injury in a severe sepsis model. These results demonstrate that the duration of rewarming in addition to the severity of sepsis should be con-
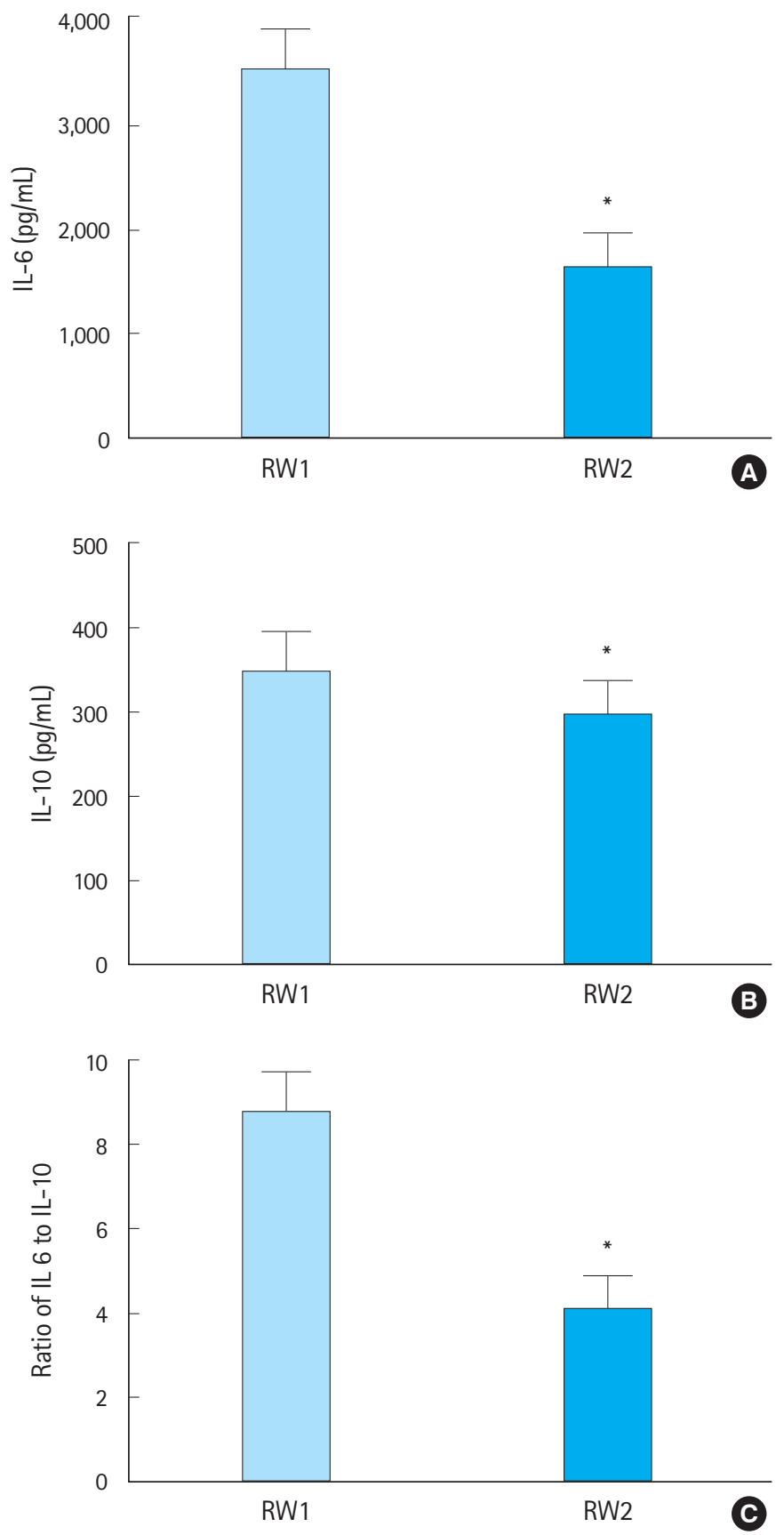

Fig. 4. Plasma interleukin (IL) concentrations. (A) Plasma IL-6 concentration is higher in the RW1 group than in the RW2 group. (B) Plasma IL-10 concentration is not significantly different between the RW1 and RW2 groups. (C) The ratio of IL-6 to IL-10 is significantly higher in the RW1 group than in the RW2 group. RW1, 1 hour of rewarming after 4 hours of therapeutic hypothermia; RW2, 2 hours of rewarming after 4 hours of therapeutic hypothermia. Values are presented as mean \pm SE. $\mathrm{n}=10$ per group. ${ }^{*} \mathrm{P}<0.05$.

sidered when applying TH to sepsis.

It has been suggested that the harmful effect of rapid rewarming is associated with ROS production and inflammatory respons- 
es. ROS have been known to play an important role in various tissue injuries. It has been reported that ROS production increased during rewarming following $\mathrm{TH}$ in hemorrhagic shock, and rapid rewarming resulted in a higher concentration of ROS in an intestinal ischemia-reperfusion model. ${ }^{5,10}$ In the present study, the MDA concentration of the RW1 group was higher than that of the RW2 group, which indicated that rapid rewarming aggravated acute lung injury by ROS-induced lipid peroxidation.

IL-6 is a proinflammatory cytokine reported to be associated with poor outcome in sepsis. ${ }^{23} \mathrm{TH}$ has reportedly decreased the concentration of IL-6, and this phenomenon has been associated with the anti-inflammatory effect of $\mathrm{TH}^{4,9,16}$ In the present study, the RW1 group demonstrated a higher concentration of IL- 6 than the RW2 group, which indicated that rapid rewarming induced the inflammatory response and was related to poor outcome in sepsis.

IL-10 is an anti-inflammatory cytokine, and few studies have analyzed the effect of rewarming after TH on IL-10. In patients with cardiac arrest and in an animal model of trauma, IL-10 concentrations did not differ between the $\mathrm{TH}$ and rewarming phases. $^{9,24}$ It has also been reported that surviving animals showed a tendency toward decreasing IL-10 concentrations after $\mathrm{TH}$ and rewarming compared to non-surviving animals in an emergency preservation and resuscitation model. ${ }^{25}$ In the present study, IL10 concentration did not differ between the RW1 and RW2 groups. The ratio of IL-6 to IL-10 represents the equilibrium of the pro- and anti-inflammatory responses, and a higher ratio of IL-6 to IL-10 was found to be correlated with poor outcome in sepsis patients. ${ }^{26}$ In the present study, the ratio of IL-6 to IL-10 was significantly higher in the RW1 group than in the RW2 group, which suggests that rapid rewarming could induce the inflammatory response.

This study has several limitations. First, we did not measure the tissue injury and plasma cytokine levels in the NT group. However, 4 of 6 rats in the NT group died within 7 hours of induction of sepsis, so measurement of tissue injury only in the surviving animals would result in a survival effect in the NT group and bias results. Second, the rewarming rates in the present study were approximately $5^{\circ} \mathrm{C} /$ hour in the RW1 group and $2.5^{\circ} \mathrm{C} /$ hour in the RW2 group, which are much faster than those currently recommended. ${ }^{6}$ As mentioned above, we previously reported that $\mathrm{TH}$ has a beneficial effect only in a severe sepsis model, and the median survival durations in the present study were 7.6 hours in the RW1 group and 9.4 hours in the RW2 group, respectively. Therefore, we could not prolong the durations of $\mathrm{TH}$ and rewarming to investigate the effect of the rewarming rate. Third, we did not use the approaches used in the clinical management of sepsis pa- tients, such as antibiotics, vasopressors, or drainage. Fourth, TH has been reported to have a beneficial effect through various mechanisms including apoptosis, immune responses, ROS, and mitochondrial function. ${ }^{27}$ Although these factors might be altered during rewarming following hypothermia, we did not assess them, with the exception of several inflammatory cytokines and MDA. Further studies are warranted to evaluate the mechanisms of the harmful effects of rapid rewarming.

In conclusion, rapid rewarming after $\mathrm{TH}$ shortens survival duration and worsens acute lung injury in sepsis, which could be associated with the inflammatory response and production of ROS.

\section{ACKNOWLEDGMENTS}

This study was partly supported by grant no. 11-2010-026 from the SNUBH Research Fund.

\section{CONFLICT OF INTEREST}

No potential conflict of interest relevant to this article was reported.

\section{REFERENCES}

1. Peberdy MA, Callaway CW, Neumar RW, et al. Part 9: postcardiac arrest care: 2010 American Heart Association Guidelines for Cardiopulmonary Resuscitation and Emergency Cardiovascular Care. Circulation 2010;122(18 Suppl 3):S768-86.

2. Polderman KH. Induced hypothermia and fever control for prevention and treatment of neurological injuries. Lancet 2008;371:1955-69.

3. Polderman KH, Herold I. Therapeutic hypothermia and controlled normothermia in the intensive care unit: practical considerations, side effects, and cooling methods. Crit Care Med 2009;37:1101-20.

4. Kim K, Kim W, Rhee JE, et al. Induced hypothermia attenuates the acute lung injury in hemorrhagic shock. J Trauma 2010; 68:373-81.

5. Kim K, Jo YH, Rhee JE, et al. Effect of speed of rewarming and administration of anti-inflammatory or anti-oxidant agents on acute lung injury in an intestinal ischemia model treated with therapeutic hypothermia. Resuscitation 2010;81:100-5.

6. Deakin CD, Morrison $\sqcup$, Morley PT, et al. Part 8: advanced life support: 2010 International Consensus on Cardiopulmonary Resuscitation and Emergency Cardiovascular Care Science with Treatment Recommendations. Resuscitation 2010;81 Suppl 1:e93-174. 
7. Alam HB, Rhee $P$, Honma $K$, et al. Does the rate of rewarming from profound hypothermic arrest influence the outcome in a swine model of lethal hemorrhage? J Trauma 2006;60:134-46.

8. Gordan ML, Kellermann K, Blobner M, Nollert G, Kochs EF, Jungwirth $\mathrm{B}$. Fast rewarming after deep hypothermic circulatory arrest in rats impairs histologic outcome and increases NFkB expression in the brain. Perfusion 2010;25:349-54.

9. Bisschops LL, Hoedemaekers CW, Mollnes TE, van der Hoeven JG. Rewarming after hypothermia after cardiac arrest shifts the inflammatory balance. Crit Care Med 2012;40:1136-42.

10. Vaagenes $P$, Gundersen $Y$, Opstad PK. Rapid rewarming after mild hypothermia accentuates the inflammatory response after acute volume controlled haemorrhage in spontaneously breathing rats. Resuscitation 2003;58:103-12.

11. Warren DE, Bickler PE, Clark JP, et al. Hypothermia and rewarming injury in hippocampal neurons involve intracellular Ca2+ and glutamate excitotoxicity. Neuroscience 2012;207: 316-25.

12. Sarcia PJ, Scumpia PO, Moldawer LL, DeMarco VG, Skimming JW. Hypothermia induces interleukin-10 and attenuates inju$r y$ in the lungs of endotoxemic rats. Shock 2003;20:41-5.

13. L'Her $E_{1}$ Amerand A, Vettier A, Sebert P. Effects of mild induced hypothermia during experimental sepsis. Crit Care Med 2006;34:2621-3.

14. Torossian $A$, Ruehlmann $S$, Middeke $M$, et al. Mild preseptic hypothermia is detrimental in rats. Crit Care Med 2004;32: 1899-903.

15. Stewart CR, Landseadel JP, Gurka MJ, Fairchild KD. Hypothermia increases interleukin-6 and interleukin-10 in juvenile endotoxemic mice. Pediatr Crit Care Med 2010;11:109-16.

16. Rim KP, Kim K, Jo YH, et al. Effect of therapeutic hypothermia according to severity of sepsis in a septic rat model. Cytokine 2012;60:755-61.

17. Lee JH, Kim K, Jo YH, et al. Therapeutic hypothermia attenuates liver injury in polymicrobial sepsis model of rats via Akt survival pathway. J Surg Res 2013;181:114-20.

18. Nishina K, Mikawa K, Takao Y, Obara H. The efficacy of fluo- rocarbon, surfactant, and their combination for improving acute lung injury induced by intratracheal acidified infant formula. Anesth Analg 2005;100:964-71.

19. Ohkawa $H$, Ohishi N, Yagi K. Assay for lipid peroxides in animal tissues by thiobarbituric acid reaction. Anal Biochem 1979;95:351-8.

20. Lavinio A, Timofeev I, Nortje J, et al. Cerebrovascular reactivity during hypothermia and rewarming. $\mathrm{Br} J$ Anaesth 2007;99: 237-44.

21. Maxwell WL, Watson $A$, Queen $R$, et al. Slow, medium, or fast re-warming following post-traumatic hypothermia therapy? An ultrastructural perspective. J Neurotrauma 2005;22:873-84.

22. Sahu $B$, Chauhan $S$, Kiran $U$, et al. Neurocognitive function in patients undergoing coronary artery bypass graft surgery with cardiopulmonary bypass: the effect of two different rewarming strategies. J Cardiothorac Vasc Anesth 2009;23:14-21.

23. Gogos CA, Drosou E, Bassaris HP, Skoutelis A. Pro- versus anti-inflammatory cytokine profile in patients with severe sepsis: a marker for prognosis and future therapeutic options. J Infect Dis 2000;181:176-80.

24. Hildebrand $F_{\text {, van }}$ Griensven $M$, Giannoudis $P$, et al. Effects of hypothermia and re-warming on the inflammatory response in a murine multiple hit model of trauma. Cytokine 2005;31: 382-93.

25. Sipos W, Duvigneau C, Sterz F, et al. Changes in interleukin-10 mRNA expression are predictive for 9-day survival of pigs in an emergency preservation and resuscitation model. Resuscitation 2010;81:603-8.

26. Taniguchi T, Koido Y, Aiboshi J, Yamashita T, Suzaki S, Kurokawa A. Change in the ratio of interleukin-6 to interleukin-10 predicts a poor outcome in patients with systemic inflammatory response syndrome. Crit Care Med 1999;27:1262-4.

27. Polderman KH. Application of therapeutic hypothermia in the ICU: opportunities and pitfalls of a promising treatment modality. Part 1: Indications and evidence. Intensive Care Med 2004;30:556-75. 